\title{
Path Planning Problem
}

\author{
Oshina Vasishth \\ ITM University \\ Gurgaon, Haryana \\ India
}

\author{
Yogita Gigras \\ ITM University \\ Gurgaon, Haryana \\ India
}

\begin{abstract}
Path planning is the way of determination of a collision free path between start and goal position through obstacles cluttered in a workspace. Though it is a complex problem, but it is an essential task for the navigation and controlling the motion of autonomous robot manipulators. This NP-complete problem (those problems is difficult to solve specially in a dynamic environment where the optimal path needs to be rerouted in real time when a new obstacle appears. This paper provides two categories of path planning approaches:Deterministic and Probabilistic approaches. Deterministic methods allow achieving the same result in each execution with the same initial conditions. They are perfectly predictable, hence suitable for static environment, but not effective when they are used in a real time environment as there could be sudden changes in environment. The most used solution to overcome the problem of real time environment are the probabilistic methods such as Particle swarm optimization[pso], Ant colony optimization[aco], genetic algorithm[ga], multi agent path planning,etc.
\end{abstract}

\section{General Terms}

Deterministic algorithm, probabilistic algorithm, aco, pso, ga, A*algorithm, dijkstra, multi agent path planning.

\section{INTRODUCTION}

Autonomous robots must act in the face of un-certainty, a direct consequence of their inability to know what the case is. When making decisions, probabilistic approaches take the root's uncertainty into account. Some consider only robot's current uncertainty other anticipate future uncertainty.

Probabilistic approaches are those which involve some degree of un-certainty in predicting their behavior and require "random variables "to describe system's components and their interactions. There is no general agreement on what"randomness" of a system actually means for example: it could be generated by change mechanism, being unpredictable, showing lack of apparent order etc.

The theory of probability is the only analytical tool available to help to map the un-predictable.

Some of the probabilistic methods used in path planning problems are ant colony optimization, particle swarm optimization, and genetic algorithm.

\section{ANT COLONY OPTIMIZATION}

The ant system was developed by Marco Dorigo and his colleagues [2]. It is inspired by the behavior of the ants; they use special chemical"pheromones" to communicate with in colonies to find optimum path between the colony and food source in an environment. This mechanism is called "stigmergy" means indirect communication among the selforganizing agents or actions.
Ant moves in random orientation from the start position and pheromones are deposited on the ground from the tail as they move around, which stimulates a natural behavioral response to the other ant group. The subsequent ants would choose a path based on amount of pheromones present on all motion paths from the start position to target. The shortest path is the greatest in intensity of its pheromones trail and vice -versa. These ants would continue reinforcing the optimization path according to the intensity of the pheromones. According to the intensity of the pheromones trail and heuristic information, the probability of the motion path is chosen by an ant. The probability is known as "transition probability" .Consider a network where ants can travel between different nodes using pheromones deposits the probability that an ant $\mathrm{k}$, when located at node $\mathrm{i}$, uses the pheromone trail $\tau_{i j}$ to compute the probability of choosing $j$ as the next node by the equation

$$
\begin{gathered}
P_{i j}^{(k)}= \begin{cases}\frac{\tau_{i j}^{\alpha}}{\sum_{j \in N_{I}^{(K)}} \tau_{i j}^{\alpha}} & \text { if } \mathrm{j} \in \mathrm{N}_{\mathrm{i}}^{(\mathrm{k})}\end{cases} \\
0 \quad \text { if } \mathrm{j} \notin \mathrm{N}_{\mathrm{i}}{ }^{(\mathrm{k})}
\end{gathered}
$$

$\mathrm{i}=$ current node

$\mathrm{j}=$ next node

$\tau_{\mathrm{ij}}=$ pheromone trail

$\alpha=$ degree of the importance of pheromones

$\mathrm{N}_{\mathrm{i}}{ }^{(\mathrm{k})}=$ indicates the set of neighbourhood nodes of ant $\mathrm{k}$ when located at node $\mathrm{i}$. The neighborhood of node $\mathrm{i}$ contain all the nodes directly connected to i except the predecessor node. This will prevent the ant from returning to same node visited immediately before nodes i.

\subsection{Path retracing and pheromones updating}

Before returning to home node, the $\mathrm{k}^{\text {th }}$ ant deposits $\Delta \tau^{\mathrm{k}}$ of the pheromone on arc it has visited. The pheromones value $\tau_{\mathrm{ij}}$ on arc (ij) traversed is updated as follows:

$$
\tau_{\mathrm{ij}} \leftarrow \tau_{\mathrm{ij}}+\Delta \tau^{\mathrm{k}}
$$

Because of increase in the pheromones, the probability of this arc being selected by the forthcoming ant will increase.

\subsection{Pheromones trail evaporation}

When an ant $\mathrm{K}$ moves to the next node, the pheromone evaporates from all arcs:-

$$
\tau_{\mathrm{ij}} \leftarrow(1-\mathrm{p}) \tau_{\mathrm{ij}}, \forall(\mathrm{i}, \mathrm{j}) \in \mathrm{A}, \text { where }
$$

$\mathrm{p} \in(0,1)$ is a parameter

$\mathrm{A}=$ segments or arc travelled by ant kin its path from home to destination. 
The decrease in pheromone intensity favors the exploration of different paths during search process.

1 iteration $=$ complete cycle $=$ pheromone evaporation + pheromone deposits.

Advantage of using Ant colony optimization is that positive feedback accounts for rapid discovery of good solutions. Positive feedback is being represented by the pheromone deposits hence if the intensity of pheromones is high for a particular node it will generally indicate that it is leading to good solutions. It can be used in dynamic applications i.e. environment which adapt changes like new distances, obstacles etc.Disadvantage of aco is difficult to analyze theoretically as ant can select randomly any path. Time for convergence is uncertain but convergence is guaranteed.

\section{PARTICLE SWARM OPTIMIZATION (PSO)}

Particle swarm optimization is a computational methodology, introduce by Dr Kennedy in 1995[5].Pso was inspired from the natural system of bird flocking, animals herds and fish schools etc.The special features observed in those social behaviors are:- producing impressive collision free and synchronized moves. When the birds are flocking for gathering food, they will move according to the sharing of information among the population. The population considered is swarm and its individuals are particles. So a swarm in pso can be identified as a set $S=\{p 1, p 2, p 3 \ldots . . p r\}$ where $p 1, p 2$, $\mathrm{p} 3, \ldots . \mathrm{pr}$ are ' $r$ ' numbers of individuals in the swarm.

Each particle in swarm is moving in the search space. It means each individual is having its own position and velocity as follow:

Set of particle position $=\left\{\mathrm{d} 1, \mathrm{~d} 2, \mathrm{~d} 3, \ldots \ldots \ldots \mathrm{d}_{\mathrm{r}}\right\}$

Set of particle velocity $=\left\{\mathrm{v} 1, \mathrm{v} 2, \mathrm{v} 3 \ldots \ldots \ldots \ldots \mathrm{v}_{\mathrm{r}}\right\}$

Each particle is having its position best value $\left(\mathrm{X}_{\text {pbest }}\right)$ based on the communicated information among the swarm, the particles will approach to one global best position .The particle having the greatest fitness is treated as the global best position $\left(\mathrm{X}_{\text {gbest }}\right)[11]$.

The particle in the swarm mutually shares their experience and they will approximate to one global best position ever visited by all particles. This process continued until they reach to destination.

This method has been successfully implemented to solve many engineering problems because of its extraordinary features like proximity, quality, diverse response, stability and adaptability. Pso is more efficient in computational view i.e. it uses less number of function evaluation than genetic algorithm. Many times pso is applied for solving scientific problems such as unknown parameters estimation in nonlinear systems [12] bioinformatics [13], machine learning [14] due to its effectiveness and faster response.

\section{GENETIC ALGORITHM}

Genetic algorithm is stochastic search techniques inspired in the natural process evolution of species guided by the principle of the survival of the fittest.

Genetic algorithms iteratively evolve a population of individuals representing candidate solutions of the optimization problem. The evolution process involves the probabilistic application of operators to find better solutions.

The solution of an optimization problem by GA starts with population random strings denoting several design vectors. The population size in ga is mutually fixed. Each string is evaluated to find its fitness values

The population is operated by 3 operators-reproduction, crossover and mutation to produce a new population of points or design. 1 cycle=reproduction +crossover+ mutation.

Reproduction: - it is first operation applied to the population to select good string from pool. The reproduction operator is also called the selection "operator" .In a commonly used reproduction operator, a string is selected from the pool with probability proportional to its fitness.

After reproduction crossover is implemented to create new strings by exchanging information among strings of mating pool

Parent $1 \mathrm{X} 1=(01011011011)$

Parent 2 X2 $=(10010111100)$

Child fitness is higher than parents.

The crossover is the main operator by which new string with better fitness values are created for new generation. So we can say that reproduction operator selects good string for mating pool, the crossover operator recombines the substring of good strings of the mating pool to create string and mutation operator alters the string locally. The use of 3 operation successfully finds new generation.

Advantage of using ga is that it do not have much mathematical requirements about the optimization problems due to their evolutionary nature [8]. The evolution operators make GA effective at performing global search.

Table 1. Comparison Table

\begin{tabular}{|c|c|c|c|}
\hline Parameter & GA & PSO & ACO \\
\hline Type of algorithm & Evolutionary & Behavioral & Behavioral \\
\hline $\begin{array}{l}\text { Theoretical } \\
\text { analysis }\end{array}$ & Easy & Difficult & Difficult \\
\hline $\begin{array}{l}\text { Information } \\
\text { sharing mechanism }\end{array}$ & $\begin{array}{l}\text { group (chromo } \\
\text { somes share } \\
\text { information } \\
\text { with each } \\
\text { other) }\end{array}$ & $\begin{array}{l}\text { one-way } \\
\text { information } \\
\text { sharing } \\
\text { mechanism(best } \\
\text { particle gives } \\
\text { out the } \\
\text { information) }\end{array}$ & $\begin{array}{l}\text { Group } \\
\text { information } \\
\text { sharing(indirect } \\
\text { communication) }\end{array}$ \\
\hline $\begin{array}{l}\text { Utilizing of } \\
\text { fitness value to } \\
\text { evaluate the } \\
\text { population }\end{array}$ & Yes & Yes & Yes \\
\hline Updating required & Yes & Yes & Yes \\
\hline Updating operators & $\begin{array}{l}\text { Yes(crossover } \\
\text { and mutation) }\end{array}$ & $\begin{array}{l}\text { No(update } \\
\text { themselves with } \\
\text { the internal } \\
\text { velocity) }\end{array}$ & $\begin{array}{l}\text { Yes(pheromone } \\
\text { s values) }\end{array}$ \\
\hline $\begin{array}{l}\text { Require ranking of } \\
\text { solutions }\end{array}$ & Yes & No & No \\
\hline $\begin{array}{l}\text { Influence of } \\
\text { population size on } \\
\text { solution time }\end{array}$ & Exponential & Linear & Linear \\
\hline $\begin{array}{l}\text { Influence of best } \\
\text { solution on } \\
\text { population }\end{array}$ & Medium & Most & Most \\
\hline $\begin{array}{l}\text { Tendency for } \\
\text { premature } \\
\text { convergence }\end{array}$ & Medium & High & High \\
\hline
\end{tabular}




\section{DETERMINISTIC SYSTEM}

It follow an entirely known rule that the state of each component and of the entire system can be given at any time in past and future .The states of deterministic systems can be described by statements or by number specifying example: Physical characteristic of the system (length, mass etc).Since they are perfectly predictable they are not very effective in a real time environment. But for some field such as avionic industry ,determinism and completeness may be two indispensible properties in order to certificate the use of some algorithms. Both these characteristics are verified by $\mathrm{A}^{*}$ algorithm.

\subsection{A* algorithm}

$\mathrm{A}^{*}$ is the most well known path finding algorithm it was developed by peter E.hart, Nils Nilsson and Bertram Raphael in 1968[17].The $\mathrm{A}^{*}$ algorithm can be considered as the best first search algorithm that combines the advantages of uniform cost and greedy searches using a fitness function.

A* algorithm equation $\mathrm{f}(\mathrm{n})=\mathrm{g}(\mathrm{n})+\mathrm{h}(\mathrm{n})$.

The term $g(n)$ denotes the accumulated cost from the start node to node $\mathrm{n}$ and $\mathrm{h}(\mathrm{n})$ is heuristic estimation of the remaining cost to get from node $n$ to the goal nodes. During the search, the $\mathrm{A}^{*}$ algorithm maintains two lists of nodes, the open list contains the nodes that have to be considered next and the closed list contains the nodes already visited. The algorithm itself consists of expanding the one from the open list whose fitness function is minimal. Expanding a node means putting it into the closed list and inserts the neighbors into the open list and evaluating the fitness function. The algorithm stops when the goal of node is expand. A* algorithm, although has been widely used because it finds the minimum cost path and it will determine the existence of free path, this conventional algorithm have a time consuming problem because it does not have a heuristic information to handle until it reach the goal. This will cause a lot of node being produce to find a short path and to avoid obstacle which will eventually make it run slower. Another deterministic algorithm is DIJKSTRA. It was proposed by E.w Dijkstrain 1959 which expose the way to find the path of minimum length between 2 nodes .Dijsktra is still one of the wellknown graph traversal algorithm.

\section{CONCLUSION}

This paper present two different approaches of path planning i.e. deterministic and probability with their advantages and drawback. The first category involves approaches in which environment modeling is necessary .These are most likely to give optimal or near optimal solutions. While the second category consider approaches in which search path may be done without modeling environment, which are suitable to deal with real-time requirement.

\section{REFERENCES}

[1] Michael BrandI, Michael MasudaI, Nicole Wehner.2010. Xiao-Hua Yu1. Ant Colony Optimization Algorithm for Robot Path Planning.
[2] M. Dorigo, V. Maniezzo \& A. Colorni.1996."Ant System: Optimization by A Colony of Cooperating Agents.

[3] N. Sariff 1 and N. Buniyamin 2 N. Sariff 1 and N. Buniyamin.2006. , An Overview of Autonomous Mobile Robot Path Planning Algorithm.

[4] Beatriz A. Garro, Humberto Sossa and Roberto A. Vázquez.2006.Path Planning Optimization Using BioInspirited Algorithms.

[5] Omar souissi, Rabie benatitallah, David duvivier, AbedlHakim artiba, Nicolas belanger and Pierre feyzeau.2013. Path Planning: A 2013 Survey .

[6] Kennedy, J., and Eberhart, R.1995. Particle Swarm Optimization.

[7] Sharad N. Kumbharana1, Prof. Gopal M. Pandey.2013.A Comparative Study of ACO, GA and SA for Solving Travelling Salesman Problem.

[8] Pengfei Guo Xuezhi Wang Yingshi Han.2010. The Enhanced Genetic Algorithms for the Optimization Design.

[9] Brendan Englot and Franz Hover .2011.Multi-Goal Feasible Path Planning Using Ant Colony Optimization.

[10] Ant Colony System Based Mobile Robot Path Planning .2010. Song-Hiang Chia, Kuo-Lan Su, Jr-Hung Guo, Cheng-Yun Chung.

[11] Jing Zhou, Guan-Zhong Dai, De-Quan He, Jun Ma, Xiao-Yan Cai.2009. Swarm Intelligence: Ant-based Robot Path Planning.

[12] Amin Zargar Nasrollahy, Hamid Haj Seyyed Javadi.2009.Using Particle Swarm Optimization for Robot Path Planning in Dynamic Environments with Moving Obstacles and Target.

[13] B.B.V.L. Deepak, Dayal R. Parhi.2013. Target Seeking Behavior of an Intelligent Mobile Robot Using Advanced Particle Swarm Optimization.

[14] Alireza1 ALFI.2011. PSO with Adaptive Mutation and Inertia Weight andIts Application in Parameter Estimation of Dynamic Systems.

[15] Zhang, Y., Xuan, J., Benildo, G.D.L.R, Clarke, R., and Habtom, W.R.2008., Reverse engineering module networks by PSO-RNN hybrid modelling. Proceedings of international Conference on Bioinformatics\& Computational Biology.

[16] Wu, Q.2010. Car assembly line fault diagnosis based on robust wavelet SVC and PSO, Expert Systems with Applications.

[17] P. Hart, N. Nilsson, and B. Raphael.1968. A formal basis for the heuristic determination of minimum cost paths. 